\title{
Signaling Load of Hierarchical Mobile IPv6 Protocol in IPv6 Networks ${ }^{\star}$
}

\author{
Ki-Sik Kong ${ }^{1}$, Sung-Ju Roh ${ }^{2}$, and Chong-Sun Hwang ${ }^{1}$ \\ 1 Dept. of Computer Science and Engineering, Korea Univ. \\ 1, 5-Ga, Anam-Dong, Sungbuk-Gu, Seoul 136-701, Korea \\ \{kskong, hwang\}@disys.korea.ac.kr \\ 2 Core Network Development Team \\ Technology R\&D Center, LG Telecom Co., Korea. \\ sjroh@lgtel.co.kr
}

\begin{abstract}
Hierarchical Mobile IPv6 (HMIPv6) has been proposed to accommodate frequent mobility of the mobile nodes and reduce the signaling load in the Internet. Though it is being considered as an efficient local mobility management protocol, its performance may vary widely depending on the various mobility and traffic related parameters. Therefore, it is essential to investigate the effects of these parameters and conduct in-depth performance study of HMIPv6. For the analysis of HMIPv6, we present a new analytical method using the mobility model based on imbedded Markov chain and a simplistic hierarchical network model. Based on these models, we analytically derive the location update cost (i.e.,binding update cost plus binding renewal cost), packet tunnelling cost, and total signaling cost, respectively, in HMIPv6. In addition, we investigate the effects of various parameters such as the speed of a mobile node, binding lifetime, and packet arrival rate on the total signaling cost generated by a mobile node during its average MAP domain residence time. The analytical results demonstrate that the signaling load generated by HMIPv6 decreases as the speed of a mobile node and binding lifetime get larger, and its packet arrival rate gets smaller.
\end{abstract}

\section{Introduction}

Recently, the demand for wireless communications has grown tremendously. The demand for "anywhere, anytime" high-speed Internet access has been a driving force for the increasing growth and advances in wireless communication and portable devices. As a consequence, these trends have prompted research into mobility support in networking protocols.

Mobile IPv6 (MIPv6) [1] has been developed by the IETF with some new functionalities, which is based on the next generation Internet protocol. However, it still has some problems. That is, MIPv6 handles local mobility of a mobile node $(\mathrm{MN})$ in the same way as it handles global mobility. As a result, an $\mathrm{MN}$

\footnotetext{
* This work was supported by the Korea Research Foundation Grant (KRF-2003-041D00403).
} 
sends the binding update message to its Home Agent (HA) and its correspondent node $(\mathrm{CN})$ each time it changes its point-of-attachment regardless of its locality. Such an approach may cause excessive signaling traffic, especially for the MNs with relatively high mobility or long distance to their HAs or CNs. In addition, this is not scalable since the generated signaling traffic can become quite overwhelming as the number of the MNs increases.

In order to overcome these drawbacks, Hierarchical Mobile IPv6 (HMIPv6) [23] has been proposed to accommodate frequent mobility of the MNs and reduce the signaling load in the Internet. HMIPv6 introduces a new entity, the Mobility Anchor Point (MAP) which works as a proxy for the HA in a foreign network. When an MN moves into a network controlled by a new MAP, it is assigned two new CoAs: a Regional CoA on the MAP's subnet (RCoA) and an on-link address (LCoA), which is the same as used for MIPv6. When an MN moves to a new subnet within the same MAP domain, only the MAP has to be informed. Note, however, that this does not imply any change to the periodic binding update message an MN has to send to the HA and the CNs, and now an MN additionally should send it to the MAP.

Generally, the performance of IP mobility protocol is highly dependent on various mobility and traffic related parameters. Therefore, it is essential to analyze and evaluate the IP mobility protocol under the various conditions, and more in-depth study needs to be performed. There have been several researches for the performance study on the IP mobility protocols.

In [3], they present a hierarchical mobility architecture that separates local mobility from global mobility to propose a mobility management scheme that is hierarchical, flexible and scalable. But, they mainly focus on evaluating the signaling bandwidth according to the binding update emission frequency. As already introduced and studied in location management for PCS networks 4, in order to evaluate the efficiency of IP mobility management protocol, the tradeoff relationship between the location update cost and the packet tunnelling cost also has to be taken into consideration in terms of total signaling cost [5]. Nevertheless, in [3], they do not consider the extra tunnelling cost for packet delivery. When the network administrator and network designer consider the deployment of HMIPv6, they should fully understand how various mobility and traffic related parameters may have an effect on the system performance. However, they just show few of the effects and relations of various mobility and traffic related parameters.

In 6], the author investigated the performance of $\mathrm{MIP}_{\mathrm{V}} 4$ regional registration. The performance measures used are registration delay and the CPU processing overheads loaded on the agents to handle mobility of the MNs. They also do not consider the signaling cost caused by the packet tunnelling.

In [7], the authors propose an analytic model for the performance analysis of HMIPv6 in IP-based cellular networks, which is based on the random walk mobility model. Based on this model, they formulate location update cost and packet delivery cost. Then, they analyze the impact of cell residence time on the location update cost and the impact of user population on the packet deliv- 
ery cost. Though their analysis is well-defined, however, they do not take both the periodic binding update and the effect of binding lifetime into consideration, which may have much effect on the total signaling cost. In addition, their analysis about the packet delivery cost of HMIPv6 is not likely to be the pure extra signaling bandwidth consumption incurred by the packet tunnelling but the network bandwidth consumption including the data traffic as well as the signaling traffic. However, from the viewpoint of IP mobility management, the consideration of the extra signaling bandwidth consumption (not including the data traffic) occurred during the processes of the location update and the packet tunnelling should be taken into [5].

In contrast to the related literature mentioned above, we perform a detailed and in-depth study of HMIPv6 in terms of the total signaling cost. Moreover, while the previous analyses do not consider either the periodic binding update or the extra packet tunnelling, our work considers both of them for the analysis. Also, we present a new analytical method using mobility model based on imbedded Markov model and a simplistic hierarchical network model. Based on these models, we analytically derive the binding update cost, binding renewal cost, packet tunnelling cost, and total signaling cost, respectively, in HMIPv6. In addition, in terms of the total signaling cost generated by an MN during its average MAP domain residence time, we show performance evaluation by investigating the effects and relations of various parameters such as the speed of an MN, binding lifetime and its packet arrival rate.

The remainder of this paper is organized as follows. In Sect.2, we describe the user mobility model and network model for the performance analysis of HMIPv6, and then the signaling cost functions of HMIPv6 are analytically derived. In Sect.3, we investigate the results of Sect. 2 by applying various numerical examples. Finally, conclusions and future works are given in Sect.4.

\section{Analytical Modelling of Hierarchical Mobile IPv6}

In this section, we first introduce our user mobility model and network model to evaluate the performance of HMIPv6. Then, for the analysis, we derive the location update cost, packet tunnelling cost, and total signaling cost, respectively, in HMIPv6.

\subsection{User Mobility Model}

For the analysis of an MN's movement behavior, we assume a simple fluid flow mobility model. The model assumes that MNs are moving at an average speed of $v$, and their movement direction is uniformly distributed over $[0,2 \pi]$, and that all the subnets are of the same rectangular shape and size, and form together a contiguous area.

The parameters used in our user mobility model are summarized as follows.

$-\gamma$ : the border crossing rate for an MN out of a subnet 


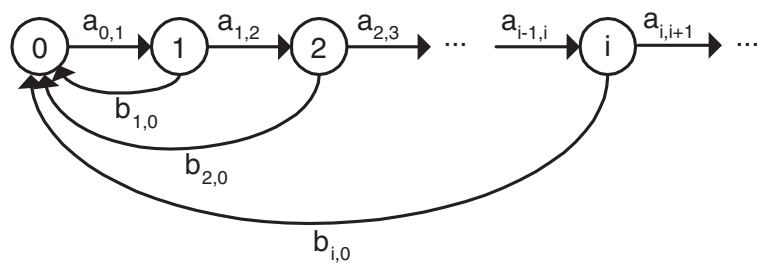

Fig. 1. State transition diagram for an imbedded Markov chain

$-\lambda$ : the border crossing rate for which an MN still stays in the same domain

$-\mu$ : the border crossing rate for an MN out of a MAP domain

From [8], the border crossing rate $\gamma$ for an $\mathrm{MN}$ out of a subnet is derived as

$$
\gamma=\frac{4 v}{\pi \sqrt{S}}
$$

where $S$ is the subnet area. We assume that a MAP domain is composed of $N$ equally large subnets. Therefore, the border crossing rate $\mu$ for an MN out of a MAP domain is

$$
\mu=\frac{4 v}{\pi \sqrt{N S}}
$$

Note that an MN that crosses a MAP domain will also cross a subnet. So, the border crossing rate $\lambda$ for which the MN still stays in the same MAP domain is obtained from Eq.(1) and (2):

$$
\lambda=\gamma-\mu=\left(1-\frac{1}{\sqrt{N}}\right) \gamma
$$

Figure 1 shows an imbedded Markov chain model, which describes the binding update process of an $\mathrm{MN}$, where $a_{i, i+1}=\lambda$ and $b_{i, 0}=\mu$. The state of an imbedded Markov chain, $i(i \geq 0)$, is defined as the number of subnets in the same MAP domain that an MN has passed by. The state transition $a_{i, i+1}(i \geq 0)$ represents an MN's movement rate to an adjacent subnet in the same MAP domain, and the state transition $b_{i, 0}(i \geq 1)$ represents an MN's movement rate (from state $i$ to state 0 ) to another subnet out of the MAP domain. We assume $\pi_{i}$ to be the equilibrium state probability of state $i$. Thus, we can obtain

$$
\begin{aligned}
& \lambda \pi_{i}=(\lambda+\mu) \pi_{i+1} \\
& \lambda \pi_{0}=\mu \sum_{i=1}^{\infty} \pi_{i}
\end{aligned}
$$

Using the Eq.(4) and (5), $\pi_{i}$ can be expressed in terms of the equilibrium state probability $\pi_{0}$ as

$$
\pi_{i}=\left(\frac{\lambda}{\lambda+\mu}\right)^{i} \pi_{0}=\left(1-\pi_{0}\right)^{i} \pi_{0}
$$




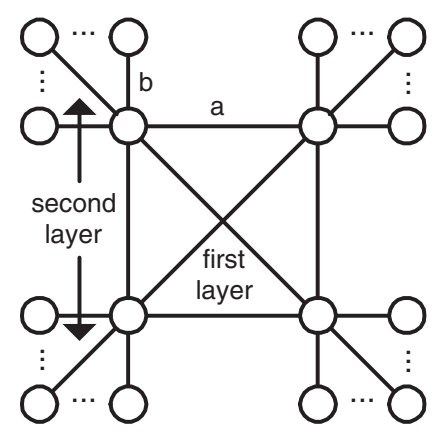

Fig. 2. Network Model

where $\pi_{0}$ is the equilibrium state probability of state 0 . By using the law of total probability, $\pi_{0}$ can be obtained as

$$
\pi_{0}=\frac{\mu}{\lambda+\mu}=1-\frac{\lambda}{\lambda+\mu}=1-\theta
$$

where $\theta=\frac{\lambda}{\lambda+\mu}$.

\subsection{Network Model}

Similar to 9], we consider a simplistic two-layer hierarchical network model given in Fig.2. The first layer has a mesh topology, which consists of $M$ nodes. Each first layer node is a root of a $N$-ary tree with depth of 1 . We assume that the HA and all the ARs are all second layer nodes, and that each MAP domain is composed of all the second layer nodes under the same first layer node. In addition, the functionality of the MAP is placed on the first layer node.

For the simplicity, the $\mathrm{CN}$, the $\mathrm{MN}$ and the HA are assumed to be located in different domain. Also, we define the domain size $(N)$ as the number of all the second layer nodes under the same MAP domain. In this model, the link hops between the first layer nodes are $a$, and the link hops between the first and second layer nodes within the same MAP domain are $b$, respectively. On the other hand, we assume that the link hops between the CN and the CN's default $\mathrm{AR}$, and the transmission cost over the wireless link are all zero.

\subsection{Cost Analysis}

In this subsection, according to the user mobility model and network model given in Sect.2.1 and 2.2, in order to evaluate the performance of HMIPv6, we analytically derive the binding update cost, binding renewal cost, packet tunnelling cost, and total signaling cost generated by an MN during its average MAP domain residence time. There are two kinds of binding update messages 
in MIPv6 and HMIPv6. That is, the one results from the MN's subnet crossing, and the other results from the expiration of the binding lifetime.

In this paper, we use binding update message to refer the former, and binding renewal message to refer the latter to differentiate these two kinds of messages. For the analysis, several parameters and assumptions mentioned in Sect.2.1 and 2.2 are used. According to our mobility model given in Sect.2.1, the average binding update cost in HMIPv6 $\left(U_{H M I P v 6}\right)$ can be formulated as

$$
\begin{aligned}
U_{H M I P v 6} & =\pi_{0}\left(U_{m}+U_{h}+\delta U_{c}\right)+U_{m} \sum_{i=1}^{\infty} i \pi_{i} \\
& =(1-\theta)\left(U_{m}+U_{h}+\delta U_{c}\right)+\frac{\theta}{1-\theta} U_{m}
\end{aligned}
$$

where $U_{m}, U_{h}$ and $U_{c}$ are the binding update costs to register with the MAP, the $\mathrm{HA}$ and the $\mathrm{CN}$, respectively. Equation $\sum_{i=1}^{\infty} i \pi_{i}$ means the average number of subnet crossing in the MAP domain, which is derived from the imbedded Markov chain in Fig.1. Based on our network model given in Sect.2.2, $U_{m}, U_{h}$ and $U_{c}$ are as follows:

$$
\begin{aligned}
U_{m} & =2 M_{B U} b \\
U_{h} & =2 M_{B U}(a+2 b) \\
U_{c} & =M_{B U}(a+2 b)
\end{aligned}
$$

where $M_{B U}$ means the bandwidth consumption caused by a binding update message. Note that the HA and the MAP must return a binding acknowledgement message, but the CNs may return it or not. For the analysis, we only consider the binding acknowledgement from the HA and the MAP, and we assume that the binding related messages are only sent alone in a separate packet without being piggybacked.

Let the binding lifetimes for the MAP, the HA and the CNs in HMIPv6 be $T_{M}, T_{H}$ and $T_{C}$, respectively. On the other hand, from the Eq.(1) and (2), the average subnet residence time and the average MAP domain residence time of an MN are $\frac{\pi \sqrt{S}}{4 v}$ and $\frac{\pi \sqrt{N S}}{4 v}$, respectively. Thus, the binding renewal rate to the MAP in HMIPv6 while an MN stays in a subnet is $\left\lfloor\frac{\pi \sqrt{S}}{4 v T_{M}}\right\rfloor$. Similarly, the binding renewal rates to the HA and the CNs in HMIPv6 while an MN stays in a MAP domain become $\left\lfloor\frac{\pi \sqrt{N S}}{4 v T_{H}}\right\rfloor$ and $\left\lfloor\frac{\pi \sqrt{N S}}{4 v T_{C}}\right\rfloor$, respectively.

On the other hand, for the calculation of the signaling costs generated to perform location update with the $\mathrm{CNs}$, we roughly define ratio of average binding time for the CNs to an MN's average MAP domain residence time, $\delta$ as the following:

$$
\delta=\frac{\sum_{i=1}^{n} C_{i}}{n \Delta}
$$

where $C_{i}$ and $n$ represent the binding time for each $\mathrm{CN}$ and the number of the CNs recorded in the MN's binding update list during the MN's average MAP 
domain residence time, respectively. $\Delta$ represents the MN's average MAP domain residence time. Consequently, the average binding renewal cost in HMIPv6 $\left(R_{H M I P v 6}\right)$ can be formulated as follows:

$$
\begin{aligned}
R_{H M I P v 6} & =\left\{(1-\theta)+\frac{\theta}{1-\theta}\right\}\left\{\left\lfloor\frac{\pi \sqrt{S}}{4 v T_{M}}\right\rfloor U_{m}+\frac{1}{\sqrt{N}}\left(\left\lfloor\frac{\pi \sqrt{N S}}{4 v T_{H}}\right\rfloor U_{h}\right.\right. \\
& \left.\left.+\delta\left\lfloor\frac{\pi \sqrt{N S}}{4 v T_{C}}\right\rfloor U_{c}\right)\right\}
\end{aligned}
$$

Therefore, the average location update cost $\left(L_{H M I P v 6}\right)$ incurred by the binding update messages and binding renewal messages is

$$
L_{H M I P v 6}=U_{H M I P v 6}+R_{H M I P v 6}
$$

Let the probability that the $\mathrm{CN}$ has a binding cache entry for an MN be $q$. Then, the average packet tunnelling cost in HMIPv6 $\left(D_{H M I P v 6}\right)$ can be formulated as follows:

$$
D_{H M I P v 6}=\frac{\pi p \sqrt{N S}}{4 v}\left\{q D_{\text {direct }}+(1-q) D_{\text {indirect }}\right\}
$$

where $D_{\text {direct }}$ and $D_{\text {indirect }}$ are the tunnelling costs for a direct packet delivery (not intercepted by the HA) and the tunnelling cost for a packet routed indirectly through the HA in HMIPv6, respectively. And, $p$ and $q$ are the average packet arrival rate for an $\mathrm{MN}$, and the probability that the $\mathrm{CN}$ has a binding cache for an $\mathrm{MN}$, respectively.

According to our network model, $D_{\text {direct }}$ and $D_{\text {indirect }}$ are as follows:

$$
\begin{aligned}
D_{\text {direct }} & =M_{P D} b \\
D_{\text {indirect }} & =M_{P D}(a+3 b)
\end{aligned}
$$

where $M_{P D}$ represents the bandwidth consumption generated by tunnelling per packet. Finally, the total signaling cost $\left(C_{H M I P v 6}\right)$ generated by an $\mathrm{MN}$ during its average MAP domain residence time in HMIPv6 can be formulated as follows:

$$
C_{H M I P v 6}=L_{H M I P v 6}+D_{H M I P v 6}
$$

\section{$3 \quad$ Numerical Results}

In this section, based on the analytical modelling of HMIPv6 in the previous section, we investigate the effects of various parameters such as the speed of an $\mathrm{MN}$, binding lifetime and its packet arrival rate on the total signaling cost. The performance measure used is the signaling bandwidth consumption per packet multiplied by the number of link hops that the packet traverses during an MN's average MAP domain residence time (i.e., Bytes $\times$ Link hops / MAP domain residence time). 
Table 1. Parameter value

\begin{tabular}{|c||c|c|}
\hline Parameter & Type & Value \\
\hline \hline$N$ & Domain size & 64 \\
\hline$S$ & Subnet area & $5 \mathrm{~m}^{2}$ \\
\hline$q$ & Probability that the CN has a binding cache for an MN & 0.7 \\
\hline$\delta$ & Ratio of avg. binding time for CNs to avg. MAP domain residence time & 0.1 \\
\hline$a$ & Link hops between the first layer nodes & 15 \\
\hline$b$ & Link hops between the first and the second layer nodes & 3 \\
\hline$M_{B U}$ & The bandwidth consumption generated by a binding update message & 68 byte \\
\hline$M_{P D}$ & The bandwidth consumption generated by tunnelling per packet & 40 byte \\
\hline
\end{tabular}

The parameter values given in Table 1 are used as default values for the performance analysis. Most parameters used in this analysis are set to typical values found in 5,6,10. The size of a binding update message is equal to the size of an IPv6 header (40 bytes) plus the size of a binding update extension header (28 bytes), so 68 bytes. In addition, the additional bandwidth consumption caused by tunnelling per packet is equal to the size of IPv6 header, so 40 bytes. According to [1,2], we set the binding lifetimes $\left(T_{H}, T_{M}, T_{C}\right)$ in HMIPv6 to be the same, and denominated as $T$.

\subsection{Location Update Cost}

Figure 3(a) shows the effect of $v$ on the average location update cost. As already mentioned, location update cost consists of both the binding update cost generated by an MN's mobility and the periodic binding renewal cost. These results presented in Fig.3(a) show that the location update cost in HMIPv6 decreases as $v$ increases. When an MN is not moving fast, most of the signaling traffic is generated by the periodic binding renewal messages. However, as the speed of an MN increases, the periodic binding renewal messages decrease and the binding update messages generated by an MN's mobility dominate most of the signaling traffic. Note here that the location update cost in HMIPv6 remains the same when $v$ exceeds $40 \mathrm{~km} /$ hour for $T=0.2$ hour or when it exceeds $30 \mathrm{~km} /$ hour for $T=0.5$ and $T=0.8$ hour in Fig.3(a). This is due to the fact that as the speed of an MN increases, it moves to an adjacent subnet before the additional binding renewal message occurs.

Figure 3(b) shows the effect of $T$ on the location update cost. As $T$ gets larger, the location update cost decreases. Note that $T$ is relatively sensitive to a slowly moving MN. This is due to the fact that the longer an MN resides in the MAP domain, the much the periodic binding renewal messages are generated. Generally, the shorter the binding lifetime is set, the much the signaling traffic is generated. Therefore, too much short binding lifetime may result in significant signaling load throughout the networks. On the other hand, the longer the binding lifetime is set, the longer the binding cache entry size of the mobility agent gets. Thus, this may result in an increase of the binding cache lookup time and memory consumption in the mobility agent. In practice, the value of binding lifetime must be specified in the implementation of HMIPv6. Therefore, further 


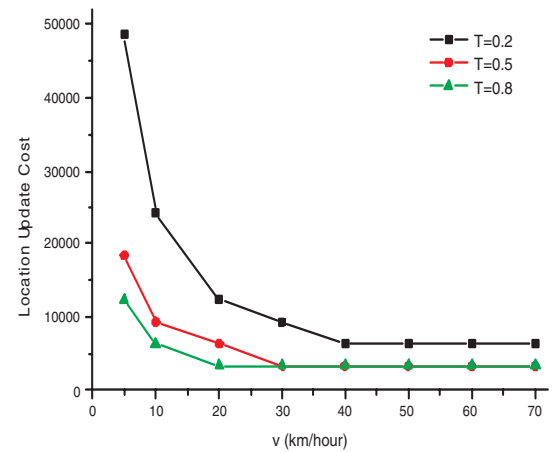

(a) Effect of $v$

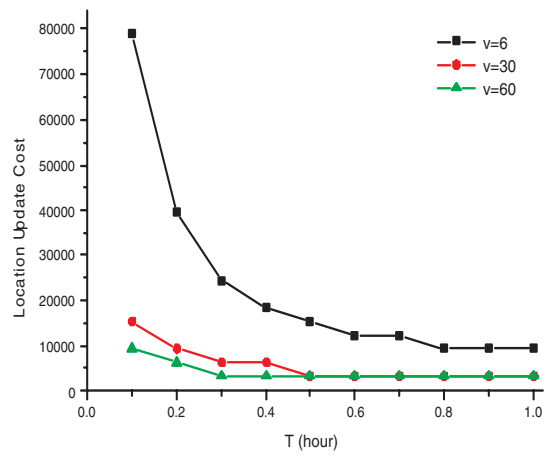

(b) Effect of $T$

Fig. 3. Location Update Cost

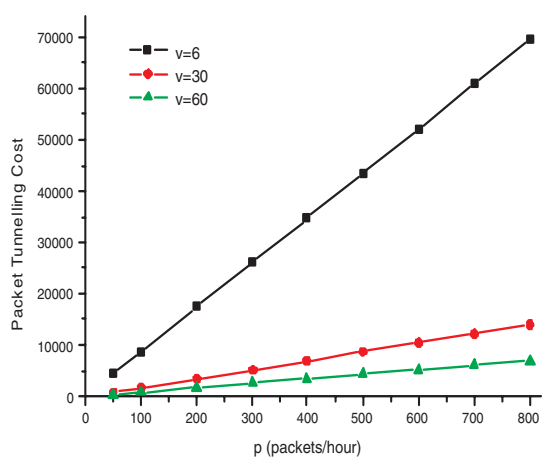

Fig. 4. Packet Tunnelling Cost

study on the effects of binding lifetime needs to be investigated to achieve the best performance.

\subsection{Packet Tunnelling Cost}

Figure 4 shows the effects of $p$ on the average packet tunnelling cost. The result shown in Fig. 4 indicates that the packet tunnelling cost is linearly increased as $p$ increases. This result also indicates that a slowly moving MN is more affected by the packet arrival rate if the packet arrival rates are the same. This is due to the fact that the MAP domain residence time of a slowly moving MN is longer than that of a fast moving MN. 


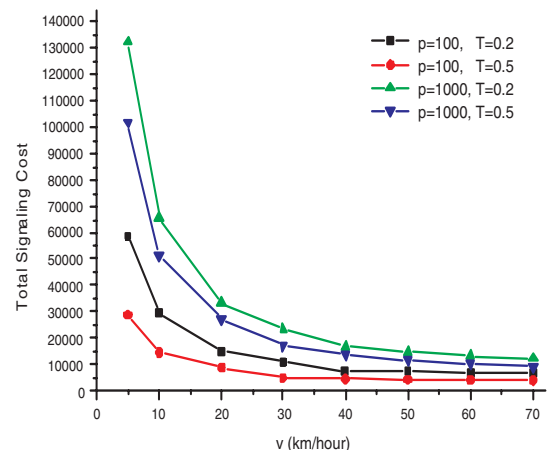

(a) Effect of $v$

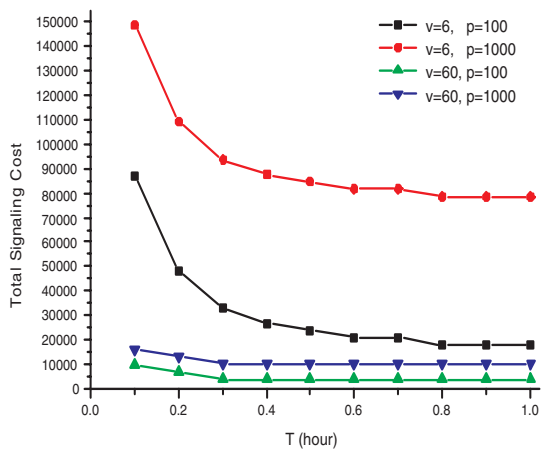

(b) Effect of $T$

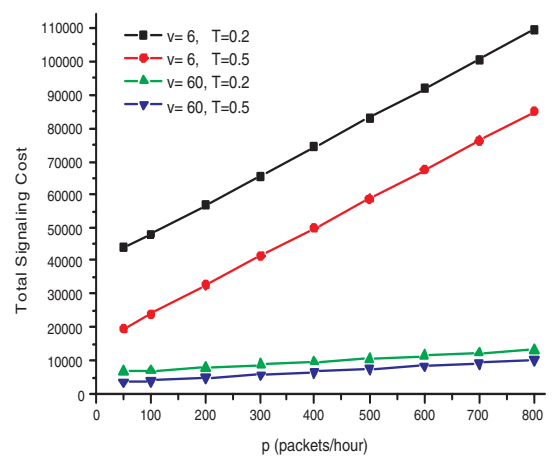

(c) Effect of $p$

Fig. 5. Total Signaling Cost

\subsection{Total Signaling Cost}

As already introduced and studied in location management for PCS networks, in order to evaluate the efficiency of IP mobility management protocol, the tradeoff relationship between the location update cost (i.e., binding update cost plus binding renewal cost) and the packet tunnelling cost also has to be taken into consideration [4]. That is, minimizing the total signaling cost of the location update cost and the packet tunnelling cost is the key focus when we evaluate and design the IP mobility protocol. In addition, it is essential to investigate the effects of various mobility and traffic related parameters from the viewpoint of the total mobility management cost.

We consider the four sets of parameters to show the variation in the total signaling costs in HMIPv6 according to the change of $v, T$, and $p$. Figure $5(\mathrm{a})$ indicates that the total signaling cost in HMIPv6 gets smaller as $v$ increases, and this phenomenon becomes more prominent as $T$ gets larger and $p$ gets smaller. Figure 5(b) indicates that the total signaling cost gets smaller as $T$ increases. As 
already mentioned in Sect.3.1, if the values of $T$ are the same, a slowly moving $\mathrm{MN}$ is relatively more affected by $T$, and a fast moving $\mathrm{MN}$ is less affected by $T$. This is because the number of binding renewal messages is proportional to the MAP domain residence time. Figure 5(c) indicates that the total signaling cost is linearly increased as $p$ increases. The results shown in Fig.5(a)-(c) can be understood from the reasons explained in Sect.3.1 and 3.2

\section{Conclusion}

In this paper, we analytically derived the signaling traffic load generated by an MN during its average MAP domain residence time when HMIPv6 is deployed. For the analysis, we presented a new analytical method using the mobility model based on imbedded Markov chain and a simplistic hierarchical network model. Then, based on these two models, we derived the binding update cost, binding renewal cost, packet tunnelling cost, and the total signaling cost, respectively, in HMIPv6. In addition, we investigated the effects of various parameters on each derived costs. The analytical results demonstrated that the signaling load of HMIPv6 decreases as the speed of an MN and binding lifetime get larger, and its packet arrival rate gets smaller.

Our future research subjects include validating our numerical results using simulation experiments. Then, we intend to extend our analytical analysis to performance comparison between the other IP mobility protocols such as MIPv6, Fast Handovers for MIPv6 (FMIPv6) and Fast Handovers for HMIPv6 (FHMIPv6), and these works are underway.

\section{References}

1. D. Johnson and C. Perkins, "Mobility Support in IPv6," draft-ietf-mobileip-ipv624.txt, June 2003.

2. H. Soliman, C. Castelluccia, K. Malki, L. Bellier, "Hierarchical Mobile IPv6 Mobility Management (HMIPv6)," draft-ietf-mipshop-hmipv6-02.txt, June 2004.

3. C. Castelluccia, "HMIPv6: A Hierarchical Mobile IPv6 Proposal," ACM Mobile Computing and Communications Review, vol.4, no.1, pp.48-59, Jan. 2000

4. I. F. Akyildiz, et al., "Mobility Management in Next-Generation Wireless Systems," Proceedings of the IEEE, Aug. 1999.

5. J. Xie, and Ian F. Akildiz, "A Novel Distributed Dynamic Location Management Scheme for Minimizing Signaling Costs in Mobile IP," IEEE Trans. on Mobilecom, vol.1, no.3, Jul-Sep. 2002.

6. M. Woo, "Performance Analysis of Mobile IP Regional Registration," IEICE Trans. on Commun, vol.E86-B, no.2, pp.472-478, Feb. 2003.

7. S. Pack and Y. Choi, "Performance Analysis of Hierarchical Mobile IPv6 in IPbased Cellular Networks," PIMRC 2003, Sep. 2003.

8. F. Baumann and I. Niemegeers, "An Evaluation of Location Management Procedures," Proc. UPC'94, pp.359-364, Sep. 1994.

9. T. Ihara, H. Ohnishi, and Y. Takagi, "Mobile IP Route Optimization Method for a Carrier-Scale IP Network," ICECCS 2000, pp.11-14 Sep. 2000.

10. R. Ramjee, K. Varadhan, L. Salgarelli, S. Thuel, W. Yuan, T. Porta, "HAWAII: A Domain-Based Approach for Supporting Mobility in Wide-Area Wireless Networks," IEEE/ACM Trans. Networking, vol.10, no.3, pp.396-410, Jun. 2002. 